\title{
Triagem nutricional: identificação de alterações nutricionais na atenção à saúde do idoso ${ }^{1}$
}

\author{
Adriane Heldt dos Santos*, Loiva Beatriz Dallepiane", Rosane Maria Kirchner"*, \\ Fhaira Petter da Silva"***, Bruna Bragagnollo Bolner"****, Aline Hert ${ }^{* * * *}$
}

\section{Resumo}

O objetivo do estudo é identificar a condição nutricional de idosos mediante a utilização de triagem, procedimento eficiente na detecção de idosos em risco, cujo cuidado é considerado prioritário na atenção à saúde geriátrica. Trata-se de um estudo do tipo quantitativo, descritivo e transversal com idosos do município de Palmeira das Missões (RS). A amostra calculada compreendeu 424 idosos selecionados aleatoriamente, considerando uma margem de erro de 5\%. A seleção desses indivíduos foi realizada por meio de sorteio aleatório dos domicílios por bairros da cidade. As variáveis analisadas foram: sociodemográfica, antropométricas e triagem nutricional. Para essa última variável, foi utilizado o instrumento denominado de Nutrition Screening
Initiative - NSI (Iniciativa de Triagem Nutricional), a fim de identificar idosos em risco nutricional. A metodologia de análise foi a estatística descritiva e o teste de Qui-Quadrado. Observou-se que, a idade variou de sessenta a 99 anos com média de 70,8 \pm 7,8 anos, e, na maioria casados, a escolaridade ficou abaixo de cinco anos e a renda de um a dois salários mínimos. A triagem identificou que $48,9 \%$ estão em bom estado nutricional, no entanto, quando somados os riscos moderado e alto, o índice ficou em $51,1 \%$, superior ao bom estado nutricional. O NSI é um instrumento de fácil aplicação, podendo contribuir como estratégia na atenção primária à saúde dos idosos.

Palavras-chave: Nutrição. Envelhecimento. Desnutrição. Saúde pública.

1 Este trabalho é parte da pesquisa denominada "Situação alimentar e nutricional da população idosa de Palmeira das Missões (RS)".

* Nutricionista, especialista em Gestão da Organização Pública em Saúde pela Universidade Federal de Santa Maria (UFSM). Endereço para correspondência: Rua Alberto Pasqualini, n 78, casa 01, Centro, São Martinho (RS), CEP: 98690-000. E-mail: adriane_heldt@hotmail.com.

** Graduada em Nutrição pela Universidāde Regional do Noroeste do Estado do Rio Grande do Sul (Unijuí). Doutora em Ciências da Saúde: Geriatria, pela Pontifícia Universidade Católica do Rio Grande do Sul (PUCRS); Docente do Centro de Educação Superior Norte do RS (CESNORS), da Universidade Federal de Santa Maria (UFSM). E-mail: loiva.dallepiane@hotmail.com.

*** Graduada em Licenciatura Plena, habilitação em Matemática, pela Universidade Regional do Noroeste do Estado do Rio Grande do Sul (Unijuí). Doutora em Engenharia Elétrica pela Pontifícia Universidade Católica do Rio de Janeiro (PUC-RJ). Docente do Centro de Educação Superior Norte do RS (CESNORS), da Universidade Federal de Santa Maria (UFSM). E-mail: rosanekirchner@gmail.com.

**** Bolsista do Programa Institucional Voluntário de Iniciação Científica (PIVIC), da Universidade Federal de Santa Maria (UFSM). Acadêmica do curso de Nutrição, Centro de Educação Superior Norte do RS (CESNORS), da Universidade Federal de Santa Maria (UFSM). E-mail: fhaira_petter@hotmail.com; brunab.bolner91@hotmail.com; alinehert@hotmail.com.

$\rightarrow$ http://dx.doi.org/10.5335/rbceh.2013.3202 


\section{Introdução}

A população idosa mundial está aumentando e, em consequência, a expe tativa de vida também mostra sinais visíveis de crescimento. As previsões populacionais indicam que em 2025 o Brasil, assim como os demais países, terá o dobro do número de pessoas idosas, e para 2050 , as estimativas para a expectativa de vida ao nascer alcançará os 85 anos. Nesse sentido, o número de indivíduos com mais de sessenta anos de idade tem apresentado um crescente e relevante aumento. A ciência da nutrição tem contribuído na saúde do ser humano, em especial, para a população idosa, pois visa melhorar o estado nutricional e a qualidade de vida dos indivíduos, proporcionando-lhes maior longevidade (SOUZA; GUARIENTO, 2009).

O processo de envelhecimento traz diferentes alterações ao ser humano. Uma dessas alterações é a própria aceitação do ciclo de vida, além das alterações fisiológicas, metabólicas, nutricionais e psicossociais, cujas mudanças influenciam diretamente nas condições de saúde e no estado nutricional do idoso (CAMPOS; MONTEIRO; ORNELAS, 2000).

Muitos são os fatores que contribuem para uma boa ou má nutrição da pessoa idosa. Dentre os quais, estão os fatores psicossociais, como a depressão e o isolamento social decorrente de diferentes motivos, como perda da audição, problemas bucais, surgimento de doenças crônicas, internação em instituições, perda de um ente querido, dificuldades financeiras, impossibilidade de realizar pequenas atividades cotidianas (OTERO et al., 2002).
A desnutrição proteica calórica (DPC) é a condição mais preocupante entre os idosos, visto que tem como consequência, maior propensão às infecções e a diminuição da qualidade de vida, podendo levar à morte. A desnutrição em idosos, com frequência e erroneamente, é considerada como o resultado do processo de envelhecimento natural (OTERO et al., 2002).

A fim de identificar idosos em situação de má nutrição, utiliza-se a triagem nutricional, a qual consiste em um parâmetro baseado em questionário simples aplicado ao idoso ou a seus familiares (ALVARENGA et al., 2010). A Associação Dietética Americana, define triagem nutricional, como o processo de identificação de características associado a problemas dietéticos ou nutricionais. Após a triagem, indivíduos em risco, devem ser submetidos à avaliação para diagnóstico nutricional e, assim, nortear as intervenções necessárias. A análise do estado nutricional pode ser realizada com a utilização de vários parâmetros, de forma isolada ou associada. Dessa forma, fica claro, que o propósito dessa triagem é identificar sujeitos em risco, enquanto a avaliação do estado nutricional permite, além do diagnóstico classificar o grau de desnutrição, servindo como fonte de informações para correções e intervenções de saúde (NAJAS; NEBULONI, 2005; BRYAN; JONES; RUSSEL, 1998).

A partir das considerações acima, emergiu a necessidade de avaliar as possíveis alterações nutricionais na saúde do idoso, por um instrumento de fácil acesso e rápida aplicação, podendo ser utilizado na gestão dos serviços de 
saúde. Assim, este estudo tem o objetivo de identificar a condição nutricional de idosos por meio da triagem, procedimento eficaz na detecção de idosos em risco nutricional, cujo cuidado é considerado prioritário na atenção à saúde geriátrica.

\section{Metodologia}

A pesquisa é quantitativa, descritiva e transversal. A população estudada é da cidade de Palmeira das Missões (RS) e com idade acima de sessenta anos. Nesse município, a população total é de 33.846 habitantes, desses, 4.037 são idosos, correspondendo a $11,93 \%$ da população. Participarm da amostra calculada 424 idosos, considerando uma margem de erro de $5 \%$, conforme Barbetta (2007). A seleção desses indivíduos, foi realizada por meio de sorteio aleatório dos domicílios, por bairros da cidade. Após a identificação do domicílio, verificou-se a disponibilidade e $o$ aceite dos idosos à pesquisa, visto que quando não havia o consentimento ao estudo, $o$ critério seguido era buscar o primeiro domicílio na sequência.

A coleta dos dados realizou-se no domicílio e foi excluídos da pesquisa os indivíduos acamados e dependentes. Os dados são oriundos do banco de dados da pesquisa denominada "Situação alimentar e nutricional da população idosa de Palmeira das Missões, RS”.

As variáveis analisadas foram: a) sociodemográfica que engloba idade, sexo, estado civil, escolaridade e renda; b) antropométricas, na qual foram aferidos o peso e a estatura com a finalidade de calcular o Índice de Massa Corporal
(IMC). O peso foi obtido utilizando-se balança portátil digital aferida de acordo com normas do Instituto Nacional de Metrologia (Inmetro) com capacidade de até $180 \mathrm{~kg}$. A balança foi posicionada em local plano e os idosos tiveram seus pesos aferidos sem sapatos, com roupas leves e sem objetos nos bolsos. A medida foi registrada em quilogramas, e a estatura em centímetros, com auxílio de estadiômetro portátil. A pessoa idosa ficou em posição ereta, com os braços estendidos para baixo e pés unidos. $\mathrm{O}$ IMC foi calculado, dividindo-se o peso em kg pelo quadrado da estatura em metros, cujo resultado foi expresso em $\mathrm{kg} / \mathrm{m}^{2}$ e classificado de acordo com os parâmetros do Nutrition Screening Initiative (NSI) (1994); c) triagem nutricional em que foi utilizado o instrumento denominado Nutrition Screening Initiative - NSI (Iniciativa de Triagem Nutricional) para identificar idosos em risco nutricional e, a partir do resultado, fornecer um escore para ser comparado com o IMC. A iniciativa dessa triagem (NSI), é uma avaliação subjetiva composta de dez questões que investiga pontos de risco para doenças, hábitos alimentares precários, perda dental ou dor de dente, dificuldade econômica, contato social reduzido, múltiplos medicamentos, perda de peso involuntária nos últimos seis meses, e a necessidade de auxílio no cuidado próprio, dentre outros. O instrumento foi desenvolvido nos EUA, em 1990, e tem como objetivo principal despertar a consciência a respeito dos problemas nutricionais, apesar da eficiência limitada para uma baixa relação de risco de 
mortalidade. $O$ resultado é apresentado com os seguintes escores: 0-2 para boa saúde nutricional; 3-5 para risco nutricional moderado; seis ou mais para risco nutricional severo.

Os dados foram processados em uma planilha eletrônica do Excel e analisados no software estatístico SPSS. A estatística descritiva que envolveu frequência, tabelas e associação de variáveis com o teste de Qui-Quadrado foi a metodologia de análise proposta por este trabalho.

A pesquisa seguiu as recomendações da Resolução n. 196/96 do Conselho Nacional de Saúde (CNS) e foi aprovada pelo Comitê de Ética em Pesquisa da Universidade Federal de Santa Maria (UFSM), sob o processo n. 23081.009908/2010-10. Os participantes da pesquisa, após a concordância, assinaram um Termo de Consentimento Livre e Esclarecido.

\section{Resultados}

Participaram da pesquisa 424 idosos, $68,4 \%$ do sexo feminino e $31,6 \%$ do sexo masculino. A idade variou entre sessenta e 99 anos, cuja média ficou em 70,8 $\pm 7,8$ anos, com predomínio da faixa etária de sessenta a 69 anos $(50,2 \%)$. Quanto ao estado civil, a maioria dos idosos é casada (53,8\%), com escolaridade abaixo de cinco anos de estudo (60,1\%), e com renda mensal de um a dois salários mínimos $(68,4 \%)$.

De acordo com a Tabela 1 , as condições que mais contribuíram para o risco nutricional foram as seguintes: doença ou condição que leva o idoso a comer tipos ou quantidades diferentes de alimentos; consumo diário de três ou mais medicamentos distintos, receitados ou automedicados; e perda ou ganho de 4,5 $\mathrm{kg}$ nos últimos seis meses.

Tabela 1 - Iniciativa de triagem nutricional (NSI) em idosos. Palmeira das Missões (RS), 2010/2011

\begin{tabular}{|c|c|c|c|}
\hline Questão & $\operatorname{Sim}_{\mathrm{n}(\%)}$ & $\begin{array}{l}\text { Não } \\
\mathrm{n}(\%)\end{array}$ & $\begin{array}{l}\text { Total } \\
\mathrm{n}(\%)\end{array}$ \\
\hline $\begin{array}{l}\text { Presença de alguma doença ou condição que faz comer tipos } \\
\text { e/ou quantidades diferentes de alimentos. }\end{array}$ & $142(33,5)$ & $281(66,3)$ & $423(99,8)$ \\
\hline Consumo de menos de duas refeições por dia. & $10(2,4)$ & $413(97,4)$ & $423(99,8)$ \\
\hline Consumo de poucas frutas/vegetais/derivados de leite ao dia. & $79(18,6)$ & $343(80,9)$ & $422(99,5)$ \\
\hline $\begin{array}{l}\text { Consumo de três ou mais doses de cerveja, vinho ou outra } \\
\text { bebida alcoólica, quase todos os dias. }\end{array}$ & $10(2,4)$ & $413(97,4)$ & $423(99,8)$ \\
\hline Presença de problemas dentários/bucais que dificultam comer. & $65(15,3)$ & $358(84,4)$ & $423(99,8)$ \\
\hline $\begin{array}{l}\text { Nem sempre tem dinheiro suficiente para comprar o alimento } \\
\text { de que necessita. }\end{array}$ & $92(21,7)$ & $330(77,8)$ & $422(99,5)$ \\
\hline Na maioria das vezes, faz as refeições sem companhia. & $65(15,3)$ & $358(84,4)$ & $423(99,8)$ \\
\hline $\begin{array}{l}\text { Consome três ou mais medicamentos diferentes todos os dias, } \\
\text { receitados ou automedicados. }\end{array}$ & $138(32,5)$ & $285(67,2)$ & $423(99,8)$ \\
\hline Perda ou ganho de 4,5 kg nos últimos seis meses. & $153(36,1)$ & $270(63,7)$ & $423(99,8)$ \\
\hline $\begin{array}{l}\text { Nem sempre se sente fisicamente capaz de comprar, } \\
\text { cozinhar e/ou alimentar-se. }\end{array}$ & $67(15,8)$ & $356(84,0)$ & $423(99,8)$ \\
\hline
\end{tabular}

Fonte: primária. 
De acordo com a Tabela 2, a maioria dos idosos encontra-se em bom estado nutricional, equivalendo a baixo risco nutricional. Ao se somar o percentual de idosos em risco nutricional moderado e alto risco, no entanto, detecta-se um total de $51,1 \%$, índice superior ao bom estado nutricional $(48,9 \%)$.

Tabela 2 - Classificação da NSI em idosos. Palmeira das Missões (RS), 2010/2011

\begin{tabular}{lcc}
\hline \multicolumn{1}{c}{ Classificação do resultado do NSI } & $\mathrm{n}$ \\
\hline Bom estado nutricional & 207 & 48,9 \\
Risco nutricional moderado & 114 & 27,0 \\
Alto risco nutricional & 102 & 24,1 \\
Total & $423^{*}$ & 99,8 \\
\hline
\end{tabular}

Fonte: primária.

Nota: * Um participante não respondeu estas questões.

Segundo a Tabela 3, quando se agrupa os participantes por sexo, e se compara o seu NSI dentro de cada categoria, observa-se comportamento similar tanto para as mulheres quanto para os homens, resultando, em sua maioria, em um bom estado nutricional. Verifica-se, porém, que ao somar os percentuais de mulheres idosas em risco moderado e alto, constatou-se como valor total um índice de $54 \%$, portanto, superior ao bom estado nutricional, que foi de $46 \%$, indicando que as mulheres apresentam maior risco nutricional.

$\mathrm{Na}$ associação entre a faixa etária e o NSI, verificou-se que apesar do bom estado nutricional ser maior em todas as categorias de idade, a partir dos setenta anos, quando se soma os riscos moderado e alto, esse valor fica superior ao bom estado nutricional (Tabela 3).

Quanto à escolaridade, verificou-se que independente dos anos de estudos os idosos, em sua maioria, classificaram-se com bom estado nutricional, no entanto, quando se soma os riscos moderado e alto, os valores ficam, novamente, superiores ao baixo risco.

Referente à renda, a maioria apresentou um NSI com bom estado nutricional para todas as categorias de renda. Para as categorias de 1 a $2 \mathrm{SM}$ e de 3 ou mais SM, no entanto, a soma dos riscos moderado e alto é superior ao de bom estado nutricional, perfazendo $52,6 \%$ e $54,3 \%$, respectivamente.

Da mesma forma, um comportamento similar foi observado na circunferência da panturrilha (CP), pois tanto quem estava com a CP baixa ou normal apresentou NSI de bom estado nutricional. Quando, porém, somou-se os riscos moderado e alto, os valores foram superiores ao de bom estado nutricional, ou seja, $52 \%$ e $50,9 \%$ abaixo e normal, respectivamente.

Ao relacionarmos o IMC com o NSI, observou-se que, independente do IMC a maioria estava em bom estado nutricional. No entanto, quando se fez o exercício de somar o risco moderado e alto, esse valor foi superior ao de bom estado nu- 
tricional, tanto para idosos com o IMC classificado como magreza, e excesso de peso (Tabela 3).

A pesquisa mostra, enfim, que o NSI e o IMC são dois instrumentos utilizados para avaliar a condição nutricional de idosos. Entretanto, o NSI tem o obje- tivo de identificar idosos em risco, não associando o excesso de peso como mau estado de nutrição em sua forma original. Ao se juntar, porém, os dois riscos nutricionais (moderado e alto), observa-se que o NSI identifica a magreza e o excesso de peso como risco nutricional.

Tabela 3 - Associação do NSI com sexo, idade, escolaridade, renda, circunferência da cintura e índice de massa corporal em idosos. Palmeira das Missões (RS), 2010/2011

\begin{tabular}{|c|c|c|c|c|c|}
\hline \multirow{2}{*}{\multicolumn{2}{|c|}{ Variável }} & \multicolumn{4}{|c|}{ NSI } \\
\hline & & \multirow{2}{*}{$\begin{array}{r}\begin{array}{c}\text { Bom estado } \\
\text { nutricional } n(\%)\end{array} \\
133(46,0)\end{array}$} & \multirow{2}{*}{$\begin{array}{l}\text { Moderado risco } \\
\text { nutricional } \mathrm{n}(\%) \\
84(29,1)\end{array}$} & \multirow{2}{*}{$\begin{array}{r}\begin{array}{c}\text { Alto risco } \\
\text { nutricional } n(\%)\end{array} \\
72(24,9)\end{array}$} & \multirow{2}{*}{$\begin{array}{l}\text { Total } n(\%) \\
289(100,0)\end{array}$} \\
\hline Sexo & Feminino & & & & \\
\hline & Masculino & $74(55,2)$ & $30(22,4)$ & $30(22,4)$ & $134(100,0)$ \\
\hline & Total & $207(48,9)$ & $114(27,0)$ & $102(24,1)$ & $423(100,0)$ \\
\hline \multirow[t]{4}{*}{ Faixa etária } & 60170 & $110(51,6)$ & $53(24,9)$ & $50(23,5)$ & $213(100,0)$ \\
\hline & 80 & $65(45,1)$ & $37(25,7)$ & $42(29,2)$ & $144(100,0)$ \\
\hline & 80 ou mais & $32(48,5)$ & $24(36,4)$ & $10(15,1)$ & $66(100,0)$ \\
\hline & Total & $207(48,9)$ & $114(27,0)$ & $102(24,1)$ & $423(100,0)$ \\
\hline \multirow[t]{3}{*}{ Escolaridade } & $<5$ anos & $125(49,0)$ & $66(25,9)$ & $64(25,1)$ & $255(100,0)$ \\
\hline & $\geq 5$ anos & $82(48,8)$ & $48(28,6)$ & $38(22,6)$ & $168(100,0)$ \\
\hline & Total & $207(48,9)$ & $114(34,0)$ & $102(24,1)$ & $423(100,0)$ \\
\hline \multirow[t]{5}{*}{ Renda } & $<1 \mathrm{SM}$ & $26(54,2)$ & $9(18,7)$ & $13(27,1)$ & $48(100,0)$ \\
\hline & $1 / 2 \mathrm{SM}$ & $137(47,4)$ & $81(28,0)$ & $71(24,6)$ & $289(100,0)$ \\
\hline & $2 \tilde{3} 3 \mathrm{SM}$ & $28(54,9)$ & $10(19,6)$ & $13(25,5)$ & $51(100,0)$ \\
\hline & 3 ou mais & $16(45,7)$ & $14(40,0)$ & $5(14,3)$ & $35(100,0)$ \\
\hline & Total & $207(48,9)$ & $114(26,9)$ & $102(24,1)$ & $423(100,0)$ \\
\hline \multirow[t]{3}{*}{ CP } & Abaixo & $36(48,0)$ & $19(25,3)$ & $20(26,7)$ & $75(100,0)$ \\
\hline & Normal & $171(49,1)$ & $95(27,3)$ & $82(23,6)$ & $348(100,0)$ \\
\hline & Total & $207(48,9)$ & $114(27,0)$ & $102(24,1)$ & $423(100,0)$ \\
\hline \multirow[t]{4}{*}{ IMC } & Magreza & $20(41,7)$ & $13(27,1)$ & $15(31,2)$ & $48(100,0)$ \\
\hline & Eutrofia & $86(54,4)$ & $40(25,3)$ & $32(20,3)$ & $158(100,0)$ \\
\hline & Excesso & $101(46,5)$ & $61(28,1)$ & $55(25,4)$ & $217(100,0)$ \\
\hline & Total & $207(48,9)$ & $114(27,0)$ & $102(24,1)$ & $423(100,0)$ \\
\hline
\end{tabular}

Fonte: primária.

Nota: $\mathrm{Na}$ análise, cada categoria das variáveis correspondeu a 100\%; $\mathrm{CP}=$ Circunferência da panturrilha; IMC= Índice de Massa Corporal. 


\section{Discussão}

A presente pesquisa investigou as características sociodemográficas e hábitos de vida dos idosos do município de Palmeira das Missões(RS). Segundo o Instituto Brasileiro de Geografia e Estatística - IBGE (2007), nessa cidade, a população idosa feminina é maior que a masculina, condição denominada de "feminilização do envelhecimento" a exemplo do que ocorre com a população idosa mundial. A mulher está propensa à menor exposição a determinados fatores de risco, como trabalho agressivo e hostil, menor consumo de cigarro e álcool, e costuma adotar atitudes para se prevenir das doenças e incapacidades (VIEIRAS, 2007). Alguns estudos mostram, que em determinadas regiões, o percentual de mulheres com mais de setenta anos está crescendo em relação às outras faixas etárias (CHAIMOWICZ, 1997).

Para que o indivíduo realize suas atividades diárias, o organismo necessita estar em bom estado nutricional. Nesse sentido, é importante que lhe seja fornecida adequada alimentação, favorecendo o desenvolvimento natural do processo de envelhecimento, fazendo com que o sujeito consiga realizar as atividades do seu cotidiano (RODRIGUES; ABOURIHAN; YAMANE, 2010).

Para avaliar o estado nutricional do idoso, utiliza-se uma associação de indicadores, considerando as limitações de cada método (SAMPAIO, 2004). Recomenda-se, para tanto, a utilização da triagem nutricional por ser considerado um método de fácil aplicação, simples, barato, que proporciona rápido e específico diagnóstico.
Durante um estudo que avaliou o risco nutricional de uma população idosa de Dourados (MS) foi encontrada classificação de $30,2 \%$ para baixo risco (correspondendo ao bom estado nutricional), $36,6 \%$ para risco moderado e $33,2 \%$ para alto risco. Os resultados indicam que, comparando o risco nutricional dos idosos de Palmeira das Missões(RS) com os de Dourados(MS), tem-se um risco de $51 \%$ e de $69,8 \%$, respectivamente (ALVARENGA et al., 2010).

Estudo semelhante foi realizado no município de Passo Fundo (RS), denominado "Projeto Passo Fundo/RS", em que $55,6 \%$ de idosos foram classificados com baixo risco (bom estado nutricional), $27,8 \%$ com risco moderado e $16,6 \%$ com alto risco (STOBBE et al., 2005). Os resultados divergem nos três estudos citados, constatando que em Passo Fundo,os idosos estão em melhor estado nutricional , uma vez que o risco nutricional, moderado e alto representaram $44,4 \%$ dos idosos.

Em Singapura, o NSI foi utilizado para determinar o risco nutricional de 2.605 indivíduos com idade entre 55 e 98 anos, encontrando-se $25,5 \%$ com risco moderado e $4,6 \%$ com alto risco (YAP; NITI, 2007).

$\mathrm{O}$ instrumento de triagem NSI pode ser utilizado pela equipe de saúde para detectar alterações do estado nutricional de uma população de idosos. $\mathrm{O}$ índice identifica, rapidamente, mudanças na ingestão alimentar decorrente da presença de doença, número de refeições, ingestão de bebida alcoólica e de alimentos, medicação, independência para aquisição e preparo de alimentos, dificuldades eco- 
nômicas e sociais, bem como, condições de saúde bucal e alteração rápida de peso corpóreo. A determinação do risco nutricional pelo NSI, porém, não substitui as medidas antropométricas e, pelo fato de ser um inquérito, poderá haver omissão de informações, ocorrendo possibilidade de erro na interpretação dos resultados (ALVARENGA et al., 2010).

Estudos relatam, contudo, que o IMC em idosos pode ser uma medida que apresenta dificuldades na sua aferição, pois no processo de envelhecimento a estatura tende a diminuir, havendo dificuldades em manter o equilíbrio postural, ou seja, ficar em pé durante algum tempo. Depreende-se, daí, que o resultado dessa medida pode não ser tão fidedigno (DAL BOSCO, 2010).

Uma ferramenta de triagem nutricional, de fácil aplicação e recomendação, exige técnicas específicas e com qualidade, como a possibilidade de elevado número de profissionais de saúde, rapidez na aplicação, baixo custo e capacidade de detecção de risco nutricional (KYLE; GENTON; PICHARD, 2005).

Em 1991, foi publicado um questionário com dez perguntas autoaplicáveis - o Nutrition Screening Initiative (NSI), com a finalidade de identificar indivíduos com idade acima de 65 anos e em risco nutricional. Esse questionário pode ser aplicado na atenção primária de saúde, com o propósito de detectar problemas relacionados à nutrição (ALVARENGA, 2010). Esse instrumento foi escolhido para ser utilizado no presente estudo por ser de fácil aplicação e por ter indicação na Atenção Básica de Saúde. Convém ressaltar, porém, que se utilizou o critério de inclusão do idoso funcionalmente capaz, o que pode acarretar maior percentual de idosos em bom estado nutricional.

É importante considerar, que o problema da desnutrição continua existindo no Brasil, ainda que, diferentemente da obesidade, afete contingentes populacionais mais limitados (MONTEIRO; SOUZA; POPKIN, 2000). Muitos idosos têm a sua qualidade de vida diminuída por problemas nutricionais associados a doenças, o que aumenta a morbidade e mortalidade. Os idosos com oitenta anos ou mais têm seu estado nutricional mais debilitado, sofrendo um impacto negativo devido à perda da massa magra e da magreza. $\mathrm{O}$ baixo IMC, associado com enfermidades pulmonares obstrutivas e tuberculose, diminui ainda mais a qualidade de vida desses indivíduos (WHO, 1995).

É recomendável, que os idosos apresentem um IMC entre 22 a $27 \mathrm{~kg} / \mathrm{m}^{2}$, utilizado como critério de baixo peso o ponto de corte de $22 \mathrm{~kg} / \mathrm{m}^{2}$ e o IMC superior a $27 \mathrm{~kg} / \mathrm{m}^{2}$ para sobrepeso (NSI, 1994; BRASIL, 2004).

Para uma melhor qualidade de vida da pessoa idosa é necessária a manutenção do peso corporal, visto que o baixo peso o torna vulnerável a infecções, porém, quando em sobrepeso, o risco é com as doenças crônicas não transmissíveis (SOUZA; GALANTE; FIGUEIREDO, 2003).

$\mathrm{O}$ uso frequente de vários tipos de medicamentos, receitados ou automedicados, está associado à idade e ao processo de envelhecimento, principalmente em virtude do aparecimento de doenças. Esses medicamentos podem influenciar na ingestão, digestão e absorção dos 
alimentos, comprometendo o seu estado nutricional, e como consequência, um estado de desnutrição (CHAIMOWICZ, 1997; MARUCCI; GOMES, 1997).

Estudos relacionam a diminuição parcial ou total de apetite em idosos com a ausência de elementos dentários e com o uso de próteses. As pessoas que usam dentaduras têm uma mastigação que varia de $75 \%$ a $85 \%$ menos eficiente do que aquelas com dentes naturais. Com isso, acabam trocando a consistência da dieta por alimentos de textura mais macia, diminuindo o consumo de carnes, frutas e verduras frescas (NOGUÉS, 1995; MARSHALL et al., 2002).

Estudo realizado com a população de Bambuí, revelou que a maioria dos indivíduos na faixa dos sessenta anos ou mais, não realiza visita regular ao dentista. Ademais, dados da Pesquisa Nacional por Amostra de Domicílios (PNAD) revelam que na faixa etária idosa, foi encontrada associação da visita ao dentista no período igual ou inferior a um ano, com as variáveis idades, local de domicílio, grau de escolaridade e renda domiciliar per capita (MATOS et al., 2001).

Como limitação do estudo, entendeu-se que a participação em maior número de idosos do sexo feminino dificultou a comparação entre os gêneros, ficando, então, os dados apresentados cada qual em sua categoria. No entanto, destaca-se que a proporção de pessoas do sexo feminino, segundo o IBGE é maior que a do sexo masculino.

\section{Conclusões}

De acordo com os resultados apresentados, observa-se que entre a amostra estudada, a idade variou entre sessenta e 99 anos, com a idade média de 70,8 anos, porém, a idade que prevaleceu foi de sessenta a 69 anos. A maioria dos idosos pesquisados é casada, seguida de um número expressivo de viúvos, com escolaridade abaixo de cinco anos e renda em torno de um a dois salários mínimos.

Os resultados da pesquisa revelam que os idosos de Palmeiras das Missões (RS) estão em bom estado nutricional. Quando, porém, é somado o risco moderado e $\mathrm{o}$ alto risco nutricional, identifica-se um maior percentual de idosos em risco nutricional do que em bom estado nutricional, isso se avaliarmos, isoladamente pelo NSI. Pelo IMC, entretanto, a maioria encontra-se em excesso de peso, mas o NSI não substitui o IMC ou qualquer outra medida antropométrica, visto que o NSI serve para detectar a presença de risco de desnutrição. Além disso, em virtude de ser um método prático, de baixo custo e de fácil manuseio, pode ser aplicado por todos os membros da equipe de saúde.

A pesquisa mostrou que a triagem nutricional pode contribuir a nível primário na detecção de alterações do estado nutricional, pois ela permite a obtenção de informações da quantidade e da qualidade das refeições, consistência, presença de doenças, consumo de frutas, verduras e laticínios, uso de medicamentos, saúde bucal, condições físicas, econômicas e sociais, além de perda ou ganho involuntário de peso. 
O estudo revelou também, que apesar de os dois instrumentos - IMC e NSI de triagem nutricional - serem utilizados para avaliar a condição nutricional de idosos, o NSI tem o objetivo de identificar idosos em risco de desnutrição, não associando o excesso de peso com mau estado nutricional, quando avaliado isoladamente.

A triagem nutricional é um instrumento que, a exemplo de outros, pode ser utilizado na identificação de idosos em risco nutricional nos serviços de saúde pública. A apropriação de processos e serviços efetivos pela equipe de saúde, bem como, pelo gestor da unidade, implica em atitude propositiva no enfrentamento dos desafios postos pelo fenômeno do envelhecimento humano.

\section{Nutrition screening: identification of changes in nutritional health care of the elderly}

\section{Abstract}

The objective of the study is to identify the nutritional status of the elderly through the use of nutritional screening, efficient procedure for the detection of nutritional risk in elderly, whose care is considered a priority in the care of geriatric health. This is a study of the quantitative, descriptive and cross-sectional with elderly in the city of Palmeira das Missões (RS). Calculated sample comprised 424 randomly selected elderly, considering a margin of error of 5\%. The selection of these individuals was conducted through random sampling of households in neighborhoods. The variables analyzed were: sociodemographic, anthropometric and nutritional screening. For this last variable was used the instrument called Nutrition Screening Initiative - NSI to identify elderly in nutritional risk. The analysis methodology was descriptive statistics and Chi-Square test. It was observed that the age ranged 6099 years, mean $70.8 \pm 7.8$ years, and the majority married, schooling under five and income from one to two minimum wages. Nutritional screening identified that $48.9 \%$ are in good nutritional status, however, when combined moderate and high risk the index was $51.1 \%$, higher than the good nutritional status. The NSI is an easy administrable tool, and can contribute as a strategy for primary health care of the elderly.

Keywords: Nutrition. Aging. Malnutrition. Public health.

\section{Referências}

ALVARENGA, M. R. M. et al. Avaliação do risco nutricional em idosos atendidos por equipes de saúde da família. Revista da Escola de Enfermagem da USP, São Paulo, v. 44, n. 4, p. 1046-1051, dez. 2010.

BARBETTA, A. P. Estatística aplicada às ciências sociais. 7. ed. Florianópolis: UFSC, 2007.

BRASIL. Ministério da Saúde. Coordenação Geral da Política de Alimentação e Nutrição. Orientações básicas para a coleta, processamento, análise de dados e informação em serviços de saúde para o sistema de vigilância alimentar e nutricional. Versão Preliminar. Brasília: Ministério da Saúde, 2004.

BRASIL. Ministério do Planejamento, Orçamento e Gestão. Instituto Brasileiro de Geografia e Estatística. Contagem Populacional 2007. Disponivel em: < http://www.ibge.gov. br/home/estatistica/populacao/contagem2007/ contagem.pdf>. Acesso em: 10 jan. 2010.

BRYAN, F.; JONES, J. M.; RUSSEL, L. Reliability and validity of nutrition screening tool to be used with clients with learning difficulties. Journal of Human Nutrition Dietetics, Malden, v. 11, n. 1, p. 41-50, feb. 1998.

CAMPOS, M. T. F. S.; MONTEIRO, J. B. R.; ORNELAS, A. P. R. C. Fatores que afetam 
o consumo alimentar e a nutrição do idoso. Revista Nutrição, Campinas, SP, v. 13, n. 3, p. 157-165, set./dez. 2000.

CHAIMOWICZ, F. A saúde dos idosos brasileiros às vésperas do século XXI: problemas, projeções e alternativas. Revista de Saúde Pública, São Paulo, v. 31, n. 2, p. 184-200, abr. 1997.

DAL BOSCO, S. M. Nutrição da mulher: uma abordagem nutricional da saúde e doença. São Paulo: Metha, 2010.

KYLE, V. G.; GENTON, L.; PICHARD, C. Hospital length of stay nutritional status. Current Opinion in Clinical Nutrition \& Metabolic Care, New York, v. 8, n. 4, p. 397402, may. 2005.

MARSHALL, T. A. et al. Oral health, nutrient intake and dietary quality in the very old. The Journal of the American Dental Associaton, Chicago, v. 133, n. 10, p. 1369-1379. oct. 2002.

MARUCCI, M. F. N.; GOMES, M. M. B. C. Interações droga-nutriente em idosos. In: PAPALÉO NETO, M. (Org.). Gerontologia. São Paulo: Atheneu, 1997. p. 273-283.

MATOS, D. L. et al. Projeto Bambuí: estudo de base populacional dos fatores associados com o uso regular de serviços odontológicos em adultos. Caderno de Saúde Pública, Rio de Janeiro, v. 7, n. 3, p. 661-668, abr. 2001.

MONTEIRO, L.; SOUZA, A. L. M.; POPKIN, B. M. Da desnutrição para a obesidade transição nutricional no Brasil. In: MONTEIRO, C. A. (Org.). Velhos e novos males da saúde no Brasil: a evolução do país e suas doenças. 2. ed. São Paulo: Hucitec, 2000. p. 247-255.

NAJAS, M.; NEBULONI, C. C. Avaliação do estado nutricional. In: RAMOS, L. R. (Org.). Guia de geriatria e gerontologia. Barueri: Manole, 2005. p. 299-314.

NOGUÉS, R. Factors que afectan la ingesta de nutrientes en elanciano y que condicionan sucorrecta nutrición. Nutrición Clínica, México, v. 15, n. 2, p. 39-44. 1995.

OTERO, V. B. et al. Mortalidade por desnutrição em idosos, região sudeste do Brasil,
1980-1997. Revista de Saúde Pública, São Paulo, v. 36, n. 2, p. 141-148, abr. 2002.

RODRIGUES, C. S.; ABOURIHAN, C. L. S.; YAMANE, R. Qualidade de vida e o estado nutricional em homens idosos institucionalizados. Cadernos da Escola de Saúde, Curitiba, n. 3, p. 1-14. 2010.

SAMPAIO, L. M. Avaliação nutricional e envelhecimento. Revista de Nutrição, Campinas, v. 17, n. 4, p. 507-514, out./dez. 2004.

SOUZA, L.; GALANTE, H.; FIGUEIREDO, D. Qualidade de vida e bem-estar dos idosos: um estudo exploratório na população portuguesa. Revista de Saúde Pública, São Paulo, v. 37, n. 3, p. 364-371, jun. 2003.

SOUZA, V. M. C.; GUARIENTO, M. E. Avaliação do idoso desnutrido. Revista Brasileira de Clínica Médica, Campinas, n. 7, p. 46-49, nov. 2009.

STOBE, J. C. et al. Projeto Passo Fundo-RS: indicadores de saúde de participantes de um grupo de terceira idade. Revista Brasileira de Ciência do Envelhecimento Humano, Passo Fundo, v. 2, n. 1, p. 89-101, jan./jun. 2005.

VIEIRAS, J. M. Hábitos dietéticos y situación nutricional de personas de edad avanzada de Rio Grande do Sul (Brasil) de três etnias diferentes. 2007. Tese (Doutorado en Ciências de La Nutrición) - Universidad Complutense de Madrid Espanha, 2007.

WORLD HEALTH ORGANIZATION. Physical status: the use and interpretation of anthropometry. WHO Technical Report Séries n. 854. Geneva, Switzerland: WHO, 1995.

YAP, K. B.; NITI, M. Nutrition screening among community-dwelling older adults in Singapore. Singapore Medical Journal, Singapore, v. 48, n. 10, p. 911-916, oct. 2007.

THE NUTRITION SCREENING INITIATIVE - NSI. Incorporating nutrition screening and interventions into medical practice a monograph for physicians. Washington, D. C. US: American Academy of Family Physicians. The American Dietetic Association. National Council on Aging Inc, 1994. 